\title{
Controlled-release carvedilol in the management of systemic hypertension and myocardial dysfunction
}

\author{
William H Frishman' \\ Linda S Henderson ${ }^{2}$ \\ Mary Ann Lukas ${ }^{2}$ \\ 'Departments of Medicine and \\ Pharmacology, New York Medical \\ College/Westchester Medical Center, \\ Valhalla, NY, USA; ${ }^{2}$ Cardiovascular \\ and Metabolic Medicine Development \\ Center, GlaxoSmithKline, Philadelphia, \\ PA, USA
}

\begin{abstract}
Cardiovascular disease is the leading cause of death worldwide. Within the treatment armamentarium, beta-blockers have demonstrated efficacy across the spectrum of cardiovascular disease - from modification of a risk factor (ie, hypertension) to treatment after an acute event (ie, myocardial infarction). Recently, the use of beta-blockers as a first-line therapy in hypertension has been called into question. Moreover, beta-blockers as a class are saddled with a misperception of having poor tolerability. However, vasodilatory beta-blockers such as carvedilol have a different hemodynamic action that provides the benefits of beta-blockade with the addition of vasodilation resulting from alpha 1-adrenergic receptor blockade. Vasodilation reduces total peripheral resistance, which may produce an overall positive effect on tolerability. Recently, a new, controlled-release carvedilol formulation has been developed that provides the clinical efficacy of carvedilol but is indicated for once-daily dosing. This review presents an overview of the clinical and pharmacologic carvedilol controlled-release data.
\end{abstract}

Keywords: beta-blockers, cardiovascular disease, heart failure, myocardial infarction, vasodilatory

\section{Beta-blockers in the treatment of cardiovascular disease}

Cardiovascular disease (CVD) is the leading cause of morbidity and mortality, accounting for $30 \%$ of all deaths worldwide (World Health Organization 2007). In the United States alone, CVD accounted for more than 36\% of all deaths in 2004 or 1 of every 2.8 deaths (American Heart Association 2007). Blood pressure and CVD risk are directly proportional. In fact, mortality from ischemic heart disease and stroke doubles for every $20-\mathrm{mmHg}$ increase in systolic blood pressure (SBP) or 10-mmHg increase in diastolic blood pressure (DBP) (Chobanian et al 2003). Furthermore, hypertension is often an antecedent to heart failure and myocardial infarction (MI). High blood pressure $(>140 / 90 \mathrm{mmHg}$ ) has been identified in approximately $69 \%$ of Americans who have suffered a first MI and $74 \%$ of patients with chronic heart failure (Rosamond et al 2008). Therefore, it is not surprising that successful treatment of hypertension has been shown to reduce the risk of stroke, coronary artery disease (CAD), and congestive heart failure, as well as overall cardiovascular morbidity and mortality (August 2003). However, optimal treatment benefits will not be observed unless patients adhere to their prescribed treatment regimens.

Adherence to prescribed medication regimens has been shown to be highest with once-daily dosing in several disease areas, and to decrease as the number of daily doses increase (Claxton et al 2001; Fonarow 2006). In fact, decreased adherence to a medication regimen was shown to contribute to up to $64 \%$ of rehospitalizations in patients with heart failure (Leventhal et al 2005; Fonarow 2006). However, tolerability 
also influences patient adherence to a medication regimen (Weber et al 2006a; Carter et al 2008).

Beta-blockers have a long history in the treatment of hypertension and cardiac dysfunction, with more than 40 years of clinical use (Frishman 2007a). However, concerns have been raised recently from hypertension metaanalyses regarding suboptimal outcomes with use of betablockers, specifically atenolol, compared with outcomes for other antihypertensive drug classes (Lindholm et al 2005; Bangalore et al 2007b). Beta-blockers have also been associated with tolerability issues and concerns regarding negative effects on glucose and lipid metabolism. However, it should be noted that not all beta-blockers are identical, as differences in mechanism of action may translate into diverse efficacy and safety profiles (Frishman 2003; Frishman 2007a).

Carvedilol is a third-generation, vasodilatory betablocker that nonselectively blocks both the beta 1- and beta 2 -adrenergic receptors and, in addition, has alpha 1-adrenergic receptor-blocking activity. Unlike traditional beta-blockers (eg, atenolol, metoprolol, and propranolol) that lower blood pressure by reducing cardiac output (Packer 1998), vasodilatory beta-blockers can lower blood pressure by reducing systemic vascular resistance (SVR) (Sundberg et al 1987). As with other beta-blockers, carvedilol has been shown to reduce sympathetic nervous system (SNS)-mediated cardiac stress and myocardial hypertrophy (Toda 2003). These activities likely contribute to the clinical benefits observed in patients treated with carvedilol for hypertension, heart failure, and post-MI left ventricular dysfunction (LVD). Moreover, the regimen of twice-daily carvedilol has been associated with a favorable side effect and tolerability profile.

In order to improve adherence to therapy and to ease the pill burden on patients, a controlled-release formulation of carvedilol (carvedilol CR) was developed and is approved for use in the same indications (ie, hypertension, heart failure, and post-MI LVD) as immediate-release (IR) carvedilol. This review presents an overview of the clinical and pharmacologic carvedilol CR data.

\section{Pharmacology of carvedilol Mechanism of action}

Traditional beta-blockers either selectively antagonize beta 1-adrenergic receptors (selective beta-blockade) or antagonize both beta 1 - and beta 2-adrenergic receptors (nonselective beta-blockade). Although beta 1-selective agents are cardioselective, selectivity is dose dependent, and at high doses beta 1-selective agents may also antagonize beta 2-adrenergic receptors (Egan et al 2005). Traditional beta-blockers reduce blood pressure primarily by decreasing cardiac output, and systemic vascular resistance is usually unchanged (Messerli et al 2004). Inhibition of norepinephrine binding to beta-adrenergic receptors results in decreased heart rate and myocyte contractility (Packer 1998).

Unlike traditional beta-blockers, carvedilol blocks norepinephrine binding to alpha 1 -adrenegric receptors as well as beta 1- and beta 2-adrenegeric receptors (Pedersen et al 2007). Alpha 1-adrenergic receptors mediate vasoconstriction. Consequently, alpha 1-blockade results in vasodilation of the peripheral arteries, decreasing SVR (Packer 1998; Fonarow 2004). In addition, preclinical evidence suggests that carvedilol can also produce nitric oxide-mediated vasodilation (Kozlovski et al 2006).

Carvedilol does not possess intrinsic sympathomimetic activity (Toda 2003). Intrinsic sympathomimetic activity induces weak stimulation of the beta-adrenergic receptors that may dampen the positive effects of beta 1-adrenergic receptor blockade (Egan et al 2005; Frishman 2007a). Of note, beta-blockers with intrinsic sympathomimetic activity have failed to demonstrate reductions in morbidity and mortality in patients with heart failure (Maack et al 2000).

Carvedilol has also demonstrated antioxidant effects possibly attributable to stimulation of endothelial nitric oxide production or reduced nitric oxide inactivation (Toda 2003). Furthermore, carvedilol may protect against reactive oxygen species (ROS) through scavenging of free radicals, suppression of free radical generation, and prevention of ferric ion-induced oxidation (Toda 2003; Dandona et al 2007). The ability of carvedilol to scavenge free radicals has been correlated with improved outcomes in patients with heart failure and a recent MI (Goldhammer et al 2007). In a study involving 39 patients with heart failure and a recent MI, carvedilol IR was shown to decrease oxygen free radical measurements using thiobarbituric acid reactive substances assay, thermochemoluminescence, and conjugated dienes methodologies in 29 patients (irrespective of dosage) after 6 months of treatment (Goldhammer et al 2007). This decrease correlated with increased heart function (6-minute walk test; baseline, $332 \mathrm{~m}$ and at 6 months, $397 \mathrm{~m} ; \mathrm{p}<0.05$ ) compared with patients who had no evidence of reduced oxygen free radical measurements (6-minute walk test; baseline $326 \mathrm{~m}$ and at 6 months, $317 \mathrm{~m} ; \mathrm{r}=0.83 ; \mathrm{p}<0.01$ ) (Goldhammer et al 2007). Moreover, patients with reduced oxygen free radical measurements had improved clinical outcomes within 1 year compared with patients who had no reduction (chronic heart failure hospital readmissions, $10 \%$ versus $50 \%$, respectively; 
$\mathrm{p}<0.01)$ and death rates (3\% versus $20 \%$, respectively; $\mathrm{p}<0.01$ ) (Goldhammer et al 2007).

Antioxidant activities decrease elevated oxidative stress, which, in turn, reduces lipid peroxidation. Reduced lipid peroxidation may contribute to protection from myocardial and brain cell ischemic death in patients with hypertension, heart failure, ischemic heart disease, diabetes, and renal dysfunction (Maggi et al 1996; Giugliano et al 1997; Moreno et al 1998; Nakamura et al 2002; Padi et al 2002). Antioxidant activities of carvedilol may also inhibit low-density lipoprotein (LDL) oxidation, which could decrease accumulation of oxidized LDL in vessel walls (Maggi et al 1996). In patients with heart failure, free fatty acid levels become elevated (possibly from SNS hyperactivity) and the myocardial rate of fatty oxidation may increase; however, during disease progression, myocardial energy efficiency is increased through a compensatory shift from the free fatty acid substrate to glucose (Stanley et al 2005). Preliminary clinical evidence suggests that pharmacologic treatments facilitating the compensatory metabolic switch at an earlier stage of disease may provide mortality benefits (Stanley et al 2005). Indeed, carvedilol has demonstrated decreased myocardial use of free fatty acids and either increased or neutral effects on myocardial glucose use in patients with heart failure (Wallhaus et al 2001; Podbregar et al 2002; Al-Hesayen et al 2005).

Carvedilol is also known to have anti-inflammatory properties. Inflammation has been linked to the pathogenesis of heart failure and atherosclerosis (Yang et al 2004; Tatli et al 2005). In a clinical trial in patients with hypertension and diabetes, carvedilol IR (12.5 mg twice daily) reduced proinflammatory markers, including plasma C-reactive protein and monocyte chemotactic protein-1 (Dandona et al 2007). Furthermore, carvedilol has been shown to decrease serum concentrations of the inflammatory cytokines IL- 6 and TNF- $\alpha$ in patients with ischemic and nonischemic dilated cardiomyopathy (Kurum et al 2007).

In addition, carvedilol has been shown to stimulate beta-arrestin signaling (Wisler et al 2007). Beta-arrestin is involved in the desensitization process of $\beta$-receptors in response to catecholamines and is implicated in $G$ proteinmediated cardiac remodeling in heart failure (Lefkowitz et al 2006; Patel et al 2008). Recent results in animal models suggest that beta-arrestin $G$ protein-independent signaling may also occur and provide cardioprotective effects (Patel et al 2008). Furthermore, work is currently underway using beta-arrestin agonists such as carvedilol to form "super" betablockers that can turn off $G$ protein-mediated signaling of the beta-receptor but still maintain the benefits of continued $\beta$-arrestin-mediated signaling on cell survival systems (Wisler et al 2007).

\section{Pharmacokinetics and pharmacodynamics}

Carvedilol CR was developed to achieve sustained concentrations over a 24-hour period, allowing once-daily dosing. The pharmacokinetic and pharmacodynamic bioequivalence of carvedilol CR and IR was established through 2 clinical studies (Henderson et al 2006; Packer et al 2006). In a double-blind, parallel-group, crossover study, 122 patients with essential hypertension were randomized to receive either low-dose carvedilol CR (20 mg daily) or carvedilol IR (6.25 mg twice daily), high-dose carvedilol CR (80 mg once daily [initiated at $20 \mathrm{mg}$ once daily and titrated to $40 \mathrm{mg}$ and $80 \mathrm{mg}$ once daily in 1-week intervals]) or carvedilol IR (25 mg twice daily [initiated at $6.25 \mathrm{mg}$ twice daily and titrated to $12.5 \mathrm{mg}$ and $25 \mathrm{mg}$ twice daily in 1-week intervals]), or placebo (Henderson et al 2006). After 22 days of treatment, patients were crossed over to the equivalent alternate carvedilol formulation for 8 days of treatment. Patients in the placebo treatment group continued to receive placebo throughout the study. Pharmacokinetic parameters were assessed at the end of each treatment session. The pharmacodynamic endpoint was the percentage change from baseline in exercise-induced heart rate. As carvedilol is a racemic mixture of $\mathrm{R}(+)$ and $\mathrm{S}(-)$ enantiomers (Nichols et al 1989), both forms were assessed in the pharmacokinetic analysis. The pharmacokinetic profiles of both enantiomers were equivalent between carvedilol CR and IR (Figure 1) (Henderson et al 2006). In addition, both formulations maintained a reduced exercise-induced heart rate over a 24-hour period (Henderson et al 2006).

In a separate 4-week study, patients with either mild to severe heart failure or with asymptomatic post-MI LVD were treated with the carvedilol IR $(3.125,6.25,12.5$, or $25 \mathrm{mg}$ twice daily) for the first 2 weeks and then switched to carvedilol CR (10, 20, 40, or $80 \mathrm{mg}$ once daily) for 2 weeks (Packer et al 2006). Trough plasma concentration, maximum plasma concentration, and area under the curve was measured for both $\mathrm{R}(+)$ and $\mathrm{S}(-)$ enantiomers after carvedilol IR and CR treatment periods. The pharmacokinetics of carvedilol IR and $\mathrm{CR}$ were bioequivalent in patients with heart failure and post-MI with LVD. However, the median time to maximum observed plasma concentration for carvedilol CR lagged 3 hours behind that of carvedilol IR, in accordance with the prolonged-release characteristics expected in a once-daily formulation. The pharmacodynamics of carvedilol CR were 


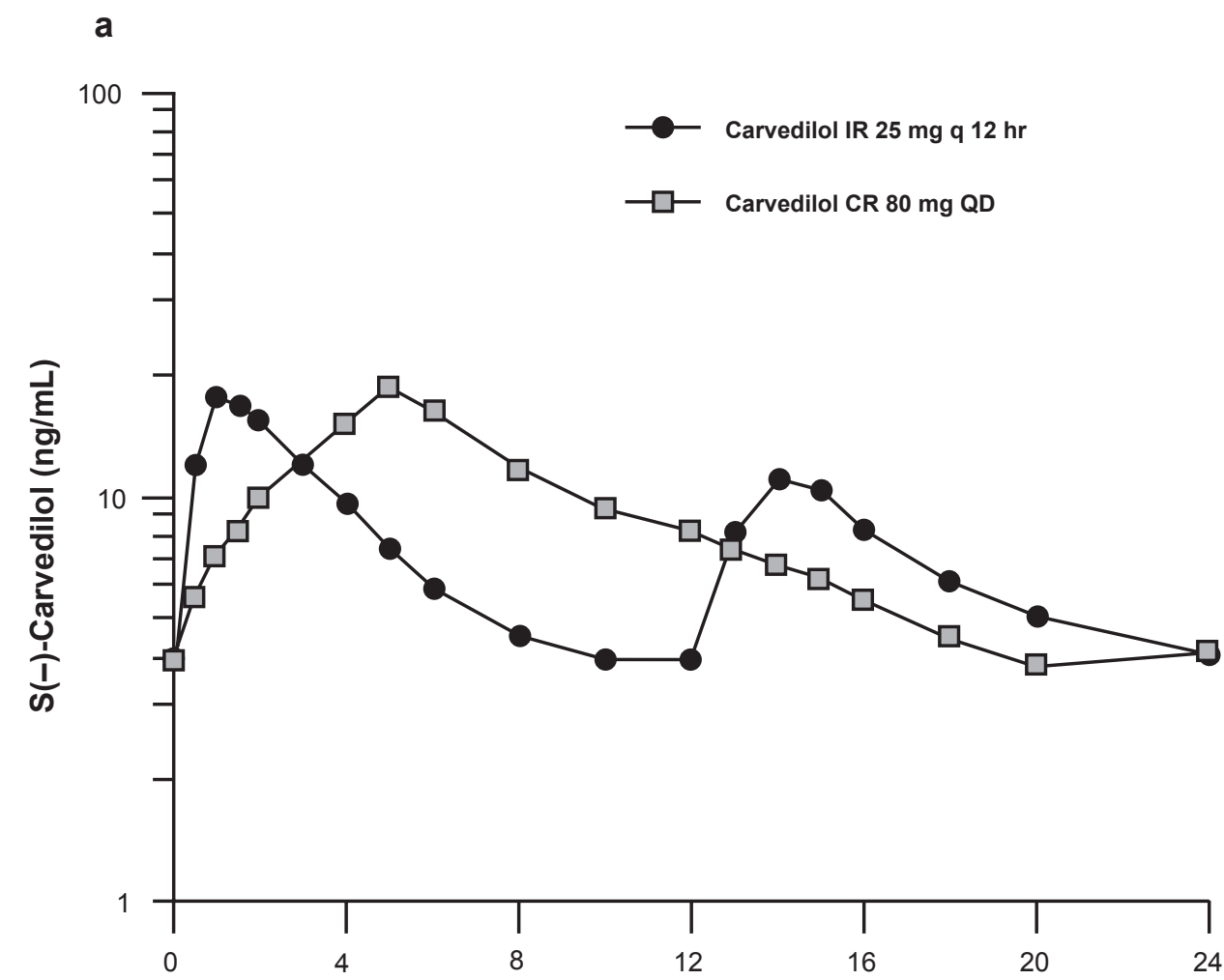

Time (hours)

b

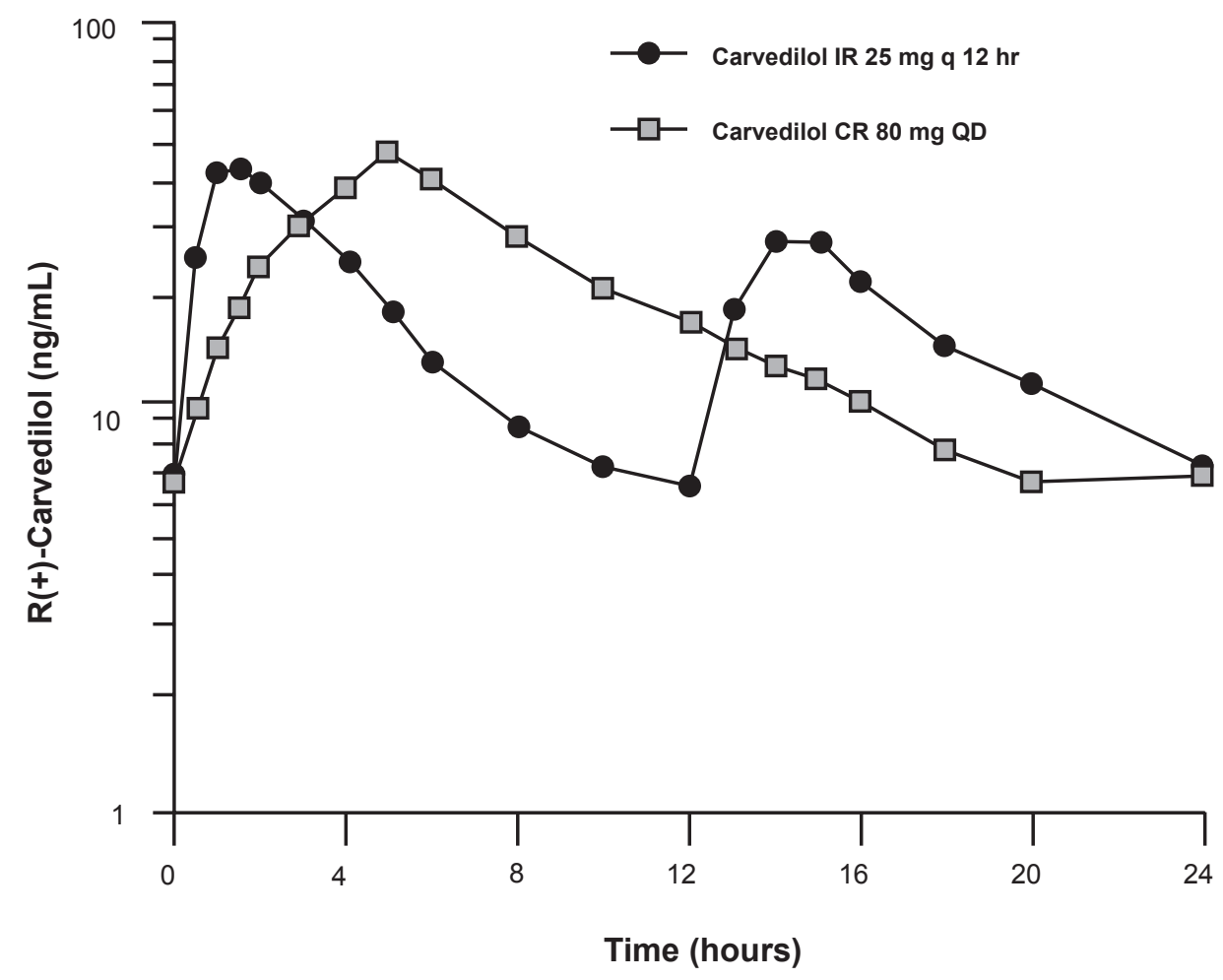

Figure I Mean steady state concentration-time profiles for $\mathrm{S}(-)$ and $\mathrm{R}(+)$ enantiomers for carvedilol immediate-release and controlled-release. Reprinted from Henderson LS, Tenero DM, Baidoo CA, et al 2006. Pharmacokinetic and pharmacodynamic comparison of controlled-release carvedilol and immediate-release carvedilol at steady state in patients with hypertension. Am J Cardiol, 98: 17L-26L. Copyright $\odot$ 2006, with permission from Elsevier.

Abbreviations: IR, immediate release, CR, controlled release, QD, once daily. 
dose proportional over the dose range tested $(10 \mathrm{mg}$ to $80 \mathrm{mg})$ (Packer et al 2006).

Notably, the bioavailability of carvedilol CR is $85 \%$ that of carvedilol IR (Tenero et al 2006). Carvedilol CR is based on carvedilol phosphate, which has a higher molecular weight than carvedilol free base and contains additional carvedilol free base compared with carvedilol IR to adjust for bioavailability. This difference contributes, in part, to slightly higher milligram dosage strengths of carvedilol CR than the "equivalent" carvedilol IR doses (Tenero et al 2006). A model of carvedilol pharmacokinetics that takes into consideration both the IR and CR formulations has been developed and performed robustly in leverage analyses (Othman et al 2007). Similar to carvedilol IR, the bioavailability and pharmacokinetics of carvedilol $\mathrm{CR}$ are influenced by food, and both formulations are recommended to be taken with food (Tenero et al 2006). However, the pharmacokinetics of carvedilol CR $40 \mathrm{mg}$ were not affected by ethanol (38 g) intake from 2 hours before to 2 hours after dosing in 39 healthy volunteers (Henderson et al 2007).

\section{Implications of mechanism of action on metabolic effects and vascular health}

Poor glycemic control predicts cardiovascular events; in particular, glycosylated hemoglobin levels linearly correlate with the risk of cardiovascular complications in patients with type 2 diabetes (Colagiuri et al 2002; Manley 2003). Moreover, dyslipidemia is a common comorbid condition in patients with hypertension (Johnson et al 2004). Men with a serum cholesterol level greater than $253 \mathrm{mg} / \mathrm{dL}$ have been shown to have a relative risk of CAD 3.8 times greater than men who had levels less than $181 \mathrm{mg} / \mathrm{dL}$ (Stamler et al 1986). Traditional, nonvasodilating beta-blockers have been associated with worsening of glycemic control and impaired lipid metabolism (Bakris et al 2006a). In contrast, because of its vasodilatory mechanism of action, which facilitates muscle uptake of glucose and increases the availability of lipoprotein lipase, carvedilol does not negatively affect glycemic control or lipid profile compared with metoprolol (Messerli et al 2004). Carvedilol CR is currently being studied in a randomized, double-blind clinical trial comparing lipid effects of metoprolol succinate extended release with carvedilol $\mathrm{CR}$ in patients with hypertension and either normal lipids or mild dyslipidemia (Bakris et al 2006b). The primary endpoints are the change from baseline in HDL-C and in triglycerides after 6 months of treatment. Several other trials have reported on glucose and lipid metabolism parameters using carvedilol IR and results have generally demonstrated improved profiles compared with metoprolol (Bakris et al 2004; Basat et al 2006; Torp-Pedersen et al 2007).

The Glycemic Effects in Diabetes Mellitus: CarvedilolMetoprolol Comparison in Hypertensives (GEMINI) trial compared the effects of carvedilol IR $(6.25 \mathrm{mg}$ to $25 \mathrm{mg}$ twice daily) with metoprolol (50 $\mathrm{mg}$ to $200 \mathrm{mg}$ twice daily) on glycemic control and lipid profile in 1235 patients with diabetes and hypertension (Bakris et al 2004). After 5 months of treatment in this randomized, double-blind, parallel-group trial, carvedilol IR did not increase $\mathrm{HbA}_{1 \mathrm{c}}(0.02 \% ; \mathrm{p}=0.65)$, whereas metoprolol significantly increased $\mathrm{HbA}_{1 \mathrm{c}}$ levels from baseline $(0.15 \% ; \mathrm{p}<0.001)$ (Figure 2$)$. Moreover, a greater number of patients withdrew because of worsening glycemic control in the metoprolol group (2.2\%) compared with the carvedilol group $(0.6 \% ; \mathrm{p}=0.04)$. These data support the results of an earlier comparison study of metoprolol and carvedilol, which found that insulin sensitivity increased with carvedilol and decreased with metoprolol treatment (Jacob et al 1996).

Comparison of lipid parameters showed that carvedilol IR decreased total serum cholesterol levels to a greater extent than metoprolol (between-group difference, $-2.9 \%$; $\mathrm{p}=0.001$ ) in the GEMINI study (Bakris et al 2004). Carvedilol IR also decreased LDL cholesterol (LDL-C) compared with metoprolol (between-group difference, $-1.3 \%$; $\mathrm{p}=0.40)$. In contrast with metoprolol, carvedilol did not significantly increase mean triglyceride levels. As kidney damage is a substantial concern for patients with hypertension and/or diabetes, kidney function was also measured in this study. Significant reductions in albumin secretion ( $16 \%$ relative reduction; $\mathrm{p}=0.003$ ) and progression to overt microalbuminuria were observed with carvedilol compared with metoprolol $(\mathrm{p}=0.04)$.

The neutral effects of carvedilol on glycemic control and lipid metabolism have also been established in patients post-MI. Fifty-nine patients post-MI were randomized to add either metoprolol (100 mg twice daily) or carvedilol IR (25 mg twice daily) to their currently prescribed regimen for 12 weeks (Basat et al 2006). After 12 weeks, patients receiving metoprolol had a significantly increased homeostasis model for insulin resistance (HOMA-IR), whereas carvedilol treatment significantly decreased HOMA-IR ( $p<0.05$ compared with baseline). In addition, carvedilol IR significantly decreased total cholesterol and LDL-C levels when compared with metoprolol $(\mathrm{p}=0.043$ and $\mathrm{p}=0.021$, respectively). 


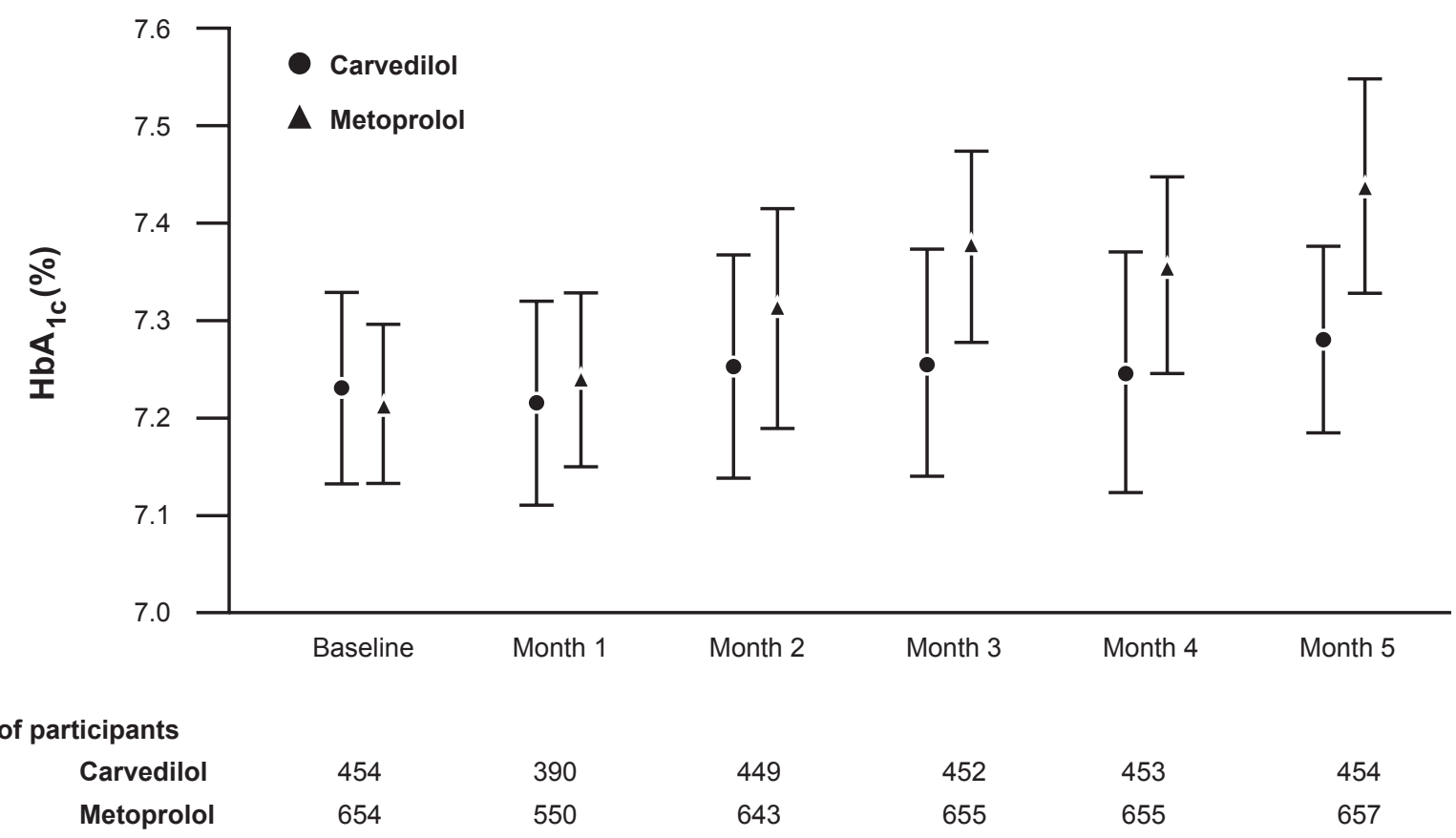

Figure 2 Glycosylated hemoglobin $\left(\mathrm{HbA}_{1}\right)$ at baseline and each maintenance month by treatment in GEMINI. Reproduced with permission from Bakris GL, Fonseca V, Katholi RE, et al 2004. Metabolic effects of carvedilol vs metoprolol in patients with type 2 diabetes mellitus and hypertension: a randomized controlled trial. JAMA,292:2227-36. Copyright (C) 2004,American Medical Association. All Rights Reserved.

Note: Data are means \pm standard deviations.

Treatment with carvedilol IR has been shown to reduce the incidence of diabetic events and new-onset diabetes among patients with heart failure. In the Carvedilol Or Metoprolol European Trial (COMET), 3029 patents with chronic heart failure were randomly assigned to treatment with target daily doses of carvedilol IR $50 \mathrm{mg}$ or metoprolol tartrate $100 \mathrm{mg}$ (Torp-Pedersen et al 2007). In a cohort of 2298 patients without diabetes at trial initiation, diabetic events (adverse events of diabetic coma, peripheral gangrene, diabetic foot, decreased glucose tolerance, or hyperglycemia) and new-onset diabetes (clinical diagnosis, repeated high random glucose level, or glucose-lowering drugs) were assessed. Over a 5-year period, fewer diabetic events were reported with carvedilol IR (122 of 1151 patients; 10.6\%) versus metoprolol (149 of 1147 patients; $13.0 \% ; \mathrm{p}=0.039$ ). New-onset diabetes (investigator reported) was also significantly less prevalent in the carvedilol IR treatment group (119 patients; 10.3\%) compared with the metoprolol treatment group (145 patients; $12.6 \% ; \mathrm{p}=0.048$ )

\section{Efficacy of carvedilol in cardiovascular disease}

The majority of clinical data for carvedilol come from studies with the IR formulation. However, several studies, both of bioequivalence and efficacy, have been conducted with carvedilol CR (Tenero et al 2006; Weber et al 2006b). Carvedilol CR is indicated for the treatment of hypertension, mild to severe heart failure, and post-MI LVD - the same indications as carvedilol IR. Data presented in this section are a mixture of carvedilol IR and CR studies.

\section{Hypertension}

The efficacy of carvedilol CR in the treatment of hypertension was established in a double-blind, parallel-group trial in which patients with essential hypertension $(\mathrm{N}=338)$ were randomized to receive carvedilol 20,40 , or $80 \mathrm{mg}$ or placebo once daily after a 4-week washout period (Weber et al 2006a; Weber et al 2006b). Change from baseline in DBP measured by 24-hour ambulatory blood pressure monitoring after 6 weeks of treatment was the primary endpoint (Weber et al 2006a). The patient population consisted of a mixture of patients, including those who were not receiving antihypertensive treatment, those with controlled hypertension (DBP $<90 \mathrm{mmHg}$ ) who were receiving antihypertensive treatment at baseline, and those with uncontrolled hypertension (DBP $\geq 90 \mathrm{mmHg}$ ) despite treatment with $\leq 2$ non-beta-blocker antihypertensive agents at baseline. Mean sitting SBP and DBP at baseline were $150 \mathrm{mmHg}$ and $99 \mathrm{mmHg}$, respectively, indicating a population with stage 1 hypertension (Chobanian et al 2003). 
Placebo-adjusted change from baseline in 24-hour DBP was $-4.03,-7.56$, and $-9.19 \mathrm{mmHg}$, respectively, with carvedilol CR 20, 40, and $80 \mathrm{mg}$ ( $\mathrm{p} \leq$ 0.001) (Weber et al 2006a). Significant reductions in placebo-adjusted 24-hour SBP were also observed $(-6.12,-9.43$, and $-11.85 \mathrm{mmHg}$, respectively) (Weber et al 2006b). Carvedilol CR treatment resulted in significant, dose-dependent reductions in peak and trough blood pressure measurements (Figure 3). Blood pressure control, defined as DBP $<90 \mathrm{mmHg}$, was achieved by $45 \%$ to $53 \%$ of patients treated with carvedilol compared with only $15 \%$ of patients who received placebo. Pulse pressure was assessed via an ad hoc analysis and was significantly reduced with carvedilol versus placebo $(\mathrm{p}<0.05)$.

These data build on the established safety and efficacy of carvedilol IR in the treatment of hypertension demonstrated in several US and international placebo-controlled trials. In patients with hypertension, carvedilol IR has been shown to reduce blood pressure and exercise and resting heart rates (Pedersen et al 2007). Blood pressure reductions are likely linked to reductions in SVR in addition to the other BP-lowering properties of all beta-blockers, which have been demonstrated in healthy volunteers treated with carvedilol IR (Sundberg et al 1987).

The antihypertensive efficacy of carvedilol has been reported in patients with hypertension and comorbid conditions, such as diabetes, which make hypertension more difficult to treat (Bakris et al 2004; Wright et al 2007). In the previously described GEMINI trial, carvedilol IR lowered SBP by $18.1 \mathrm{mmHg}$ and DBP by $9.9 \mathrm{mmHg}$, which was consistent with the $16.9 \mathrm{mmHg}$ and $9.5 \mathrm{mmHg}$ decreases in SBP and DBP, respectively, observed with metoprolol (Bakris et al 2004). At the end of the beta-blocker titration period, $37 \%$ and $36 \%$ of patients treated with carvedilol IR and metoprolol, respectively, achieved blood pressure goals $(<130 / 80 \mathrm{mmHg}$ for patients with diabetes) (Wright et al 2007). It should be noted that patients enrolled in this study were already receiving a stable regimen of antihypertensive therapy with an angiotensin-converting enzyme (ACE) inhibitor or an angiotensin-receptor blocker.

Carvedilol CR has demonstrated efficacy in the treatment of hypertension providing blood pressure control in up to $53 \%$ of the clinical patient population, which included patients with uncontrolled hypertension already receiving other classes of antihypertensive agents (Weber et al 2006a). Additionally, based on the results of trials conducted with carvedilol IR, carvedilol CR may provide an equivalent extent of efficacy in more difficult to treat patients such as those patients with hypertension and diabetes.

\section{Heart failure}

Along the cardiovascular continuum, heart failure is a natural progression of uncontrolled hypertension. A recently completed trial in patients with heart failure compared the effects of carvedilol CR with those of carvedilol IR.

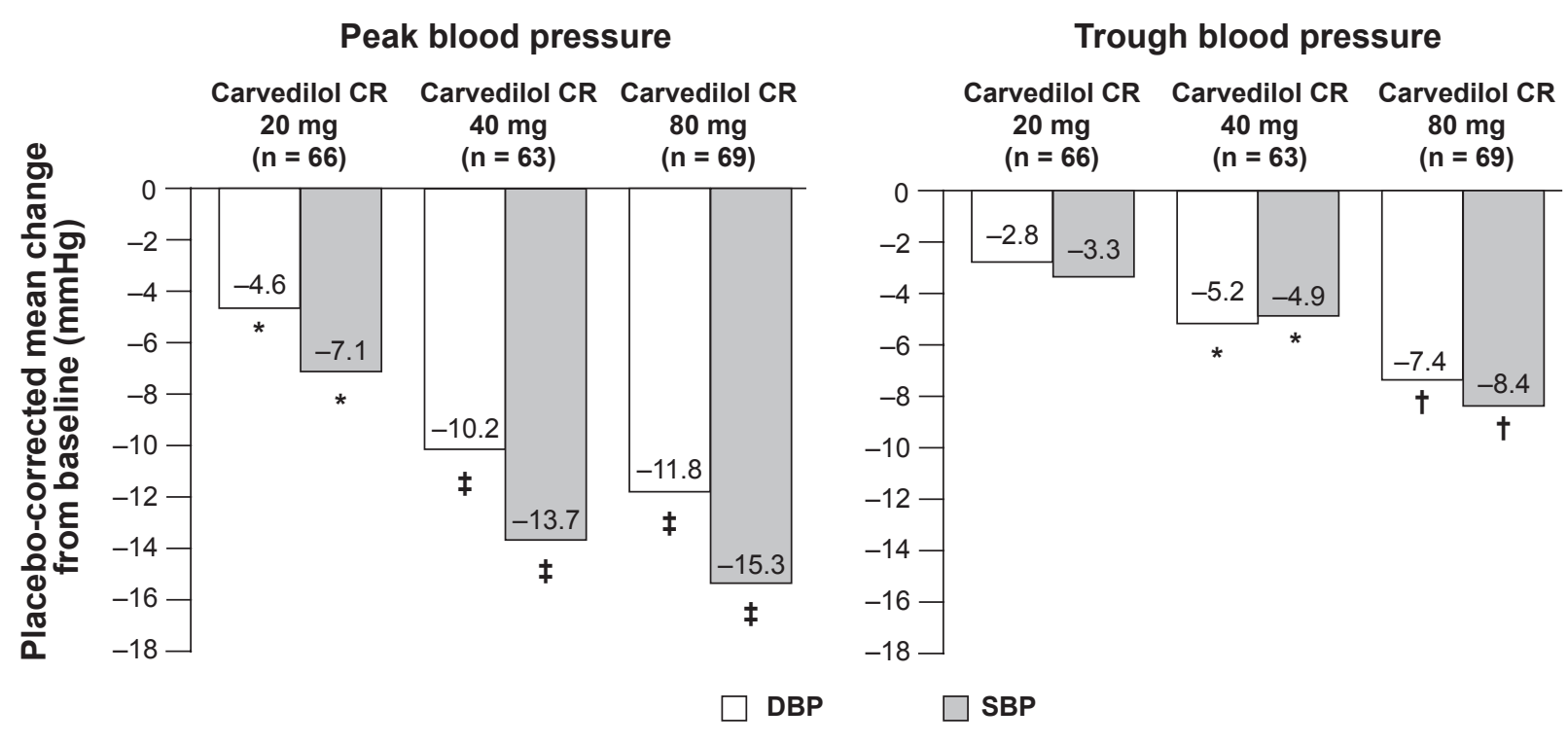

Figure 3 Controlled-release carvedilol in hypertension. Adapted from Weber MA, Sica DA,Tarka EA, et al Controlled-release carvedilol in the treatment of essential hypertension. Am J Cardiol, 98:32L-38L. Copyright (C) 2006, with permission from Elsevier.

Abbreviations: CR, controlled release, DBP, diastolic blood pressure, SBP, systolic blood pressure.

Note: Data are change from baseline in model-adjusted peak ( 3 to 7 hours) diastolic blood pressure and systolic blood pressure measured by ambulatory blood pressure monitoring. ${ }^{*} \mathrm{p}<0.05,{ }^{\dagger} \mathrm{p}<0.001,{ }^{\ddagger} \mathrm{p}<0.000$ I, based on pair-wise comparison of change from baseline with placebo. 
Coreg: A Multicenter, Randomized, Double-Blind, Double-Dummy, Parallel-Group Study to Compare Effects of Coreg CR and Coreg IR on Ejection Fraction in Subjects With Stable Heart Failure (COMPARE) trial randomized patients with mild to severe heart failure and a left ventricular ejection fraction of $40 \%(\mathrm{~N}=253)$ to either carvedilol CR (from 10 to $80 \mathrm{mg}$ once daily) or carvedilol IR (from 3.125 to $25 \mathrm{mg}$ twice daily) for up to 8 months (Greenberg et al 2006). The primary efficacy endpoint was the change from baseline in left ventricular end-systolic volume index at 6 months. Secondary endpoints included change from baseline in left ventricular remodeling and serum B-type natriuretic peptide, hospitalization for heart failure and all other causes, death from all causes, compliance, and safety and tolerability of carvedilol CR. Full results are not yet published.

The beneficial effects of carvedilol IR have been demonstrated along the spectrum of severity from mild to severe heart failure (Frishman 1998). The Australia/New Zealand (ANZ) double-blind trial randomized 415 patients with chronic, stable, mild heart failure to receive either carvedilol IR (from $3.125 \mathrm{mg}$ to $25 \mathrm{mg}$, as tolerated) or placebo twice daily for 12 months (Australia/New Zealand Heart Failure Research Collaborative Group 1997). Carvedilol IR increased left ventricular ejection fraction from baseline by $5.1 \%$ at 12 months and decreased left-ventricular and end-systolic dimensions by $1.7 \mathrm{~mm}$ and $3.2 \mathrm{~mm}$, respectively, compared with placebo. After a mean follow-up of 19 months, carvedilol IR decreased the combined risk of death or hospitalization in patients with heart failure risk by $26 \%$ versus placebo.

The US Carvedilol Heart Failure Study Group enrolled 1094 patients with mild to severe heart failure in a doubleblind, stratified program, assigning patients to 1 of 4 treatment protocols on the basis of their exercise capacity (Packer et al 1996). The mortality and hospitalization data from these 4 trials were analyzed together and considered together by the Data and Safety Monitoring Board for the trial. Patients were randomized to receive carvedilol IR (from 6.25 to $50 \mathrm{mg}$, if tolerated) or placebo twice daily and were followed for 6 months (12 months for the mild heart failure group). Compared with placebo, carvedilol IR significantly reduced mortality rates $(3.2 \%$ versus $7.8 \%$; $p<0.001)$, risk of hospitalization for cardiovascular causes $(14.1 \%$ versus $19.6 \%$; $\mathrm{p}=0.036)$, and the combined endpoint of hospitalization or death $(15.8 \%$ versus $24.6 \%$; $<0.001)$. The program was stopped early based on the significant improvements in survival with carvedilol IR compared with placebo. This finding is notable as the patients were only followed for an average of 6.5 months, which is a treatment period usually considered too short in duration to observe an effect on mortality and morbidity rates.

The Carvedilol Prospective Randomized Cumulative Survival (COPERNICUS) study randomized 2289 patients with symptoms of heart failure at rest or on minimal exertion with a left ventricular ejection fraction of $<25 \%$ (ie, severe heart failure) to receive either placebo or carvedilol IR, (from $3.125 \mathrm{mg}$ to $25 \mathrm{mg}$, as tolerated) (Packer et al 2002). Treatment with carvedilol IR resulted in a $27 \%$ reduction of the combined endpoint of death or hospitalization for a cardiovascular reason ( $\mathrm{p}<0.0001$ versus placebo), as well as a $31 \%$ reduction in the combined endpoint of death or hospitalization for heart failure ( $\mathrm{p}<0.001$ versus placebo). Carvedilol IR treatment also significantly reduced the frequency of serious adverse events, including worsening heart failure, sudden death, cardiogenic shock, and ventricular tachycardia ( $\mathrm{p}=0.002$ versus placebo).

Additionally, over a mean duration of 58 months in the COMET study, significantly fewer deaths occurred in the carvedilol group (512 of 1511 patients; 34\%) compared with the metoprolol group (600 of 1518 patients; 40\%; $\mathrm{p}=0.0017$ ) (Figure 4) (Poole-Wilson et al 2003). Cardiovascular death was less prevalent in the carvedilol group than in the metoprolol group (438 versus 534 patients; $\mathrm{p}=0.0004$ ), as were sudden death, circulatory failure, and stroke (Poole-Wilson et al 2003; Torp-Pedersen et al 2005). Results for the combined endpoint of all-cause mortality or all-cause admissions were similar in both groups $(74 \%$ and $76 \%$ of patients for carvedilol IR and metoprolol, respectively). It should be noted that metoprolol tartrate at $50 \mathrm{mg}$ twice daily has not been shown in clinical trials to provide a mortality benefit. However, of note in the metoprolol succinate heart failure trial (MERIT-HF), the mean daily dose was $159 \mathrm{mg}$ daily, which is equivalent to $106 \mathrm{mg}$ daily of metoprolol tartrate (Poole-Wilson et al 2003).

Another long-term study that compared carvedilol IR (49 mg daily) with metoprolol tartrate (124 mg daily) in patients with chronic heart failure $(\mathrm{N}=150)$ demonstrated a greater decrease in exercise heart rate with carvedilol than with metoprolol after 13 to 15 months of treatment ( $p=0.006$ ) (Metra et al 2000). In contrast, a recent trial comparing carvedilol IR (32 mg daily) and metoprolol succinate ( $96 \mathrm{mg}$ daily) in a similar patient population $(\mathrm{N}=37)$ observed a significant increase in exercise heart rate with carvedilol versus metoprolol (135 \pm 4 versus $117 \pm 6$ beats/minute, respectively; $\mathrm{p}=0.02$ ), although peak exercise norepinephrine 


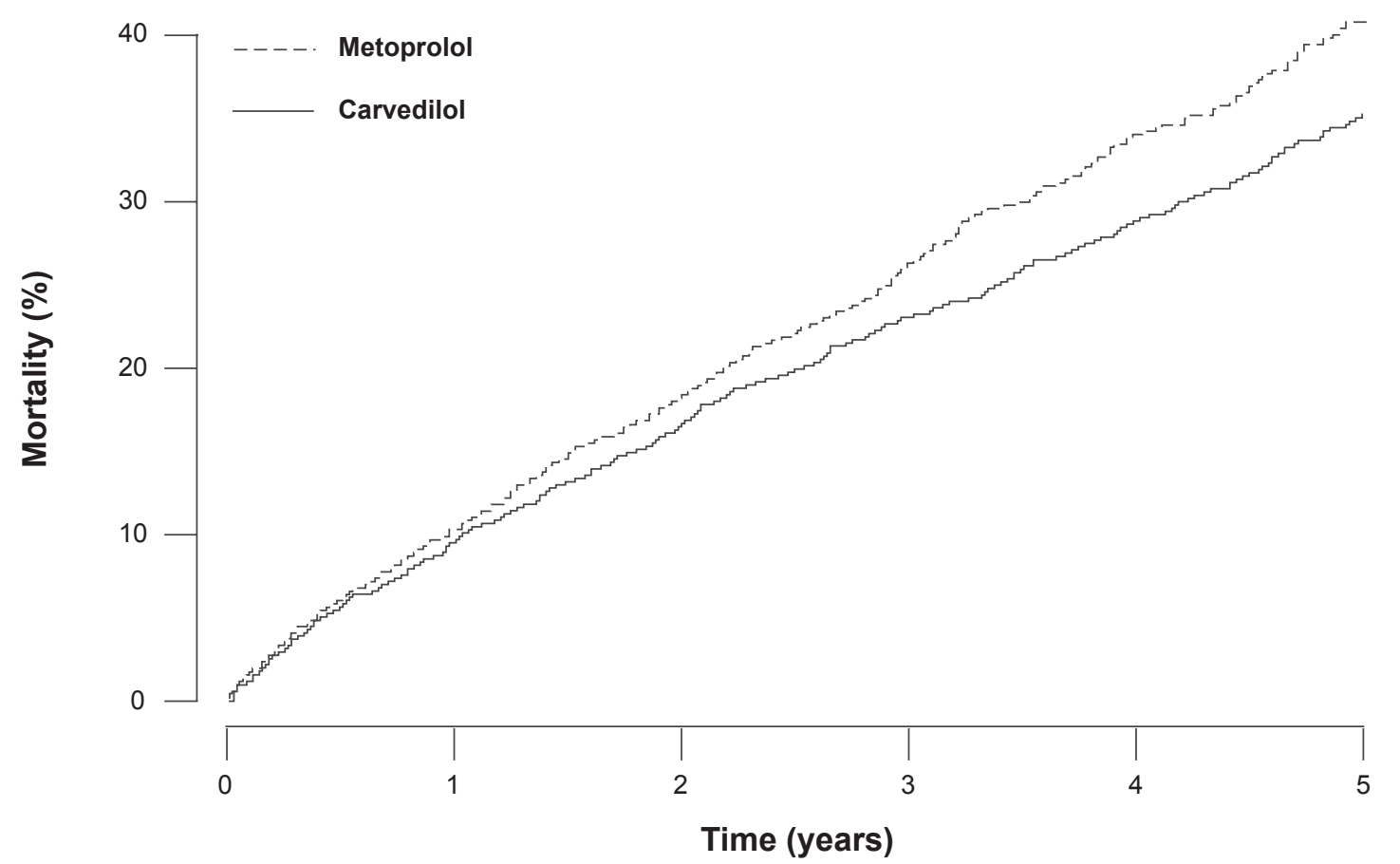

\section{Number at risk}

Carvedilol Metoprolol 1518

$\begin{array}{lll}1511 & 1366 & 1259 \\ 1518 & 1359 & 1234\end{array}$

1259
$1155 \quad 1002$

933
383

352

Figure 4 Kaplan-Meier estimates of all-cause mortality in COMET. Reprinted from Poole-Wilson PA, Swedberg K, Cleland JG, et al Comparison of carvedilol and metoprolol on clinical outcomes in patients with chronic heart failure in the Carvedilol Or Metoprolol European Trial (COMET): randomised controlled trial. Lancet, 362:7-I3. Copyright (C) 2003, with permission from Elsevier.

levels were similar (2736 \pm 320 versus $2403 \pm 372 \mathrm{pg} / \mathrm{mL}$, respectively) (Vittorio et al 2008). These findings are of note because attenuation of exercise-induced heart rate is used as a surrogate for the extent of beta 1-adrenergic blockade and possibly to evaluate dosing equivalency. Many reasons may contribute to the results of these trials, including nonequivalent doses or different formulations or durations of treatment.

\section{Post-MI LVD}

Carvedilol is currently the only beta-blocker approved for use in patients with post-MI LVD. The only clinical data available in this patient population is with carvedilol IR. The Carvedilol Post-Infarct Survival Control in LV Dysfunction (CAPRICORN) study investigated the benefits of a betablocker (carvedilol IR) in patients with post-MI LVD who were receiving standard therapy. In this double-blind trial, 1959 patients who had experienced an MI and had a left ventricular ejection fraction $\leq 40 \%$ were randomized to receive either carvedilol IR (from $6.25 \mathrm{mg}$ up to $25 \mathrm{mg}$ as tolerated) or placebo twice daily (Dargie 2001). The mean follow-up duration was 1.3 years, and $74 \%$ of patients achieved the maximum dose of carvedilol IR ( $25 \mathrm{mg}$ twice daily). In this study, carvedilol IR decreased all-cause mortality by $23 \%$ versus placebo (Figure 5). However, there was no significant difference between groups in the composite endpoint of all-cause mortality or cardiovascular hospital admission. Data from CAPRICORN showed that carvedilol IR has antiarrhythmic effects post-MI (McMurray et al 2005). Moreover, a substudy from CAPRICORN reported positive effects of carvedilol IR on ventricular remodeling (Doughty et al 2004). The authors speculate that the influence on ventricular remodeling may be a substantial contributing factor to the clinical efficacy observed in patients with post-MI LVD. More recently, Fonarow and colleagues assessed the 30-day outcomes from CAPRICORN. Reductions with carvedilol IR versus placebo were observed in mortality, fatal or nonfatal MI; the composite endpoint of death, nonfatal MI, or cardiac arrest; and the composite endpoint of all-cause mortality or nonfatal MI (Fonarow et al 2007).

Based on the CAPRICORN data, the number needed to treat to prevent 1 death for 1 year was 43 (Otterstad 


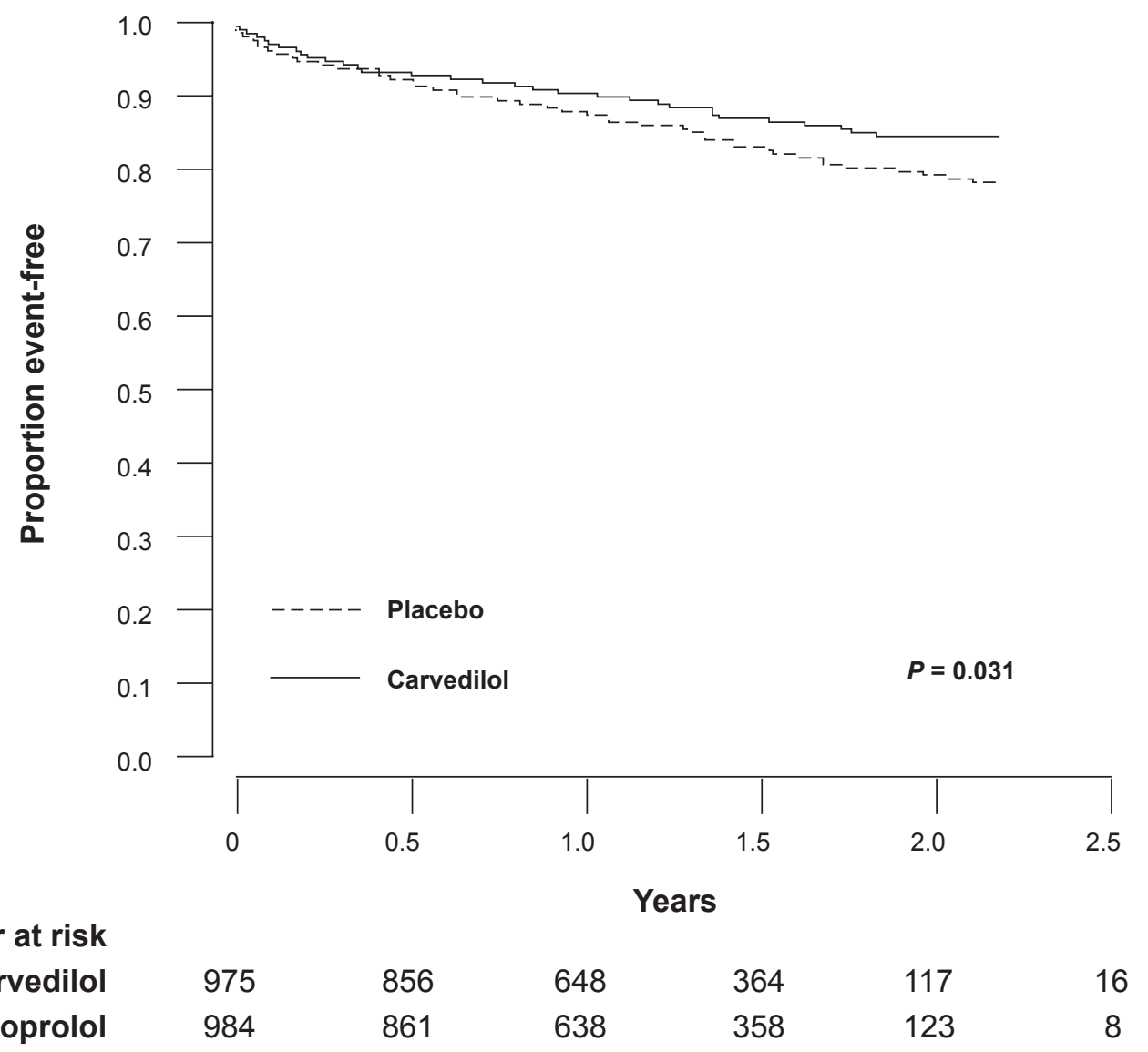

Figure 5 Kaplan-Meier estimates of event-free survival in CAPRICORN. Reprinted from Dargie HJ, for the CAPRICORN Investigators. Effect of carvedilol on outcome after myocardial infarction in patients with left-ventricular dysfunction: the CAPRICORN randomised trial. Lancet, 357:I385-90. Copyright @ 200I, with permission from Elsevier.

et al 2002). This compares favorably with data from the atenolol Beta-Blocker Heart Attack Trial (BHAT) showing that 80 patients would need treatment for 1 year to prevent 1 death (Otterstad et al 2002). In contrast, the Norwegian Timolol Trial reported that 25 patients needed treatment for 1 year to prevent 1 death (Otterstad et al 2002).

\section{Safety and tolerability of carvedilol CR}

The clinical utility of traditional beta-blockers has been limited by concerns regarding negative effects on glucose and lipid metabolism (Bakris et al 2006a; Bangalore et al 2007b) and a high incidence of side effects, including fatigue, erectile dysfunction, and weight gain (Bangalore et al 2007b). Unlike traditional beta-blockers, the alpha 1-blocking vasodilatory activity with carvedilol does not interfere with glucose and lipid metabolism (discussed previously) and has a positive influence on its tolerability profile.

Carvedilol CR is generally well tolerated across its therapeutic indications (Henderson et al 2006; Packer et al 2006; Weber et al 2006a). Adverse events reported in clinical trials were consistent with the mechanism of action of carvedilol and the health status of the patient population under evaluation. In the bioequivalence study in patients with hypertension $(\mathrm{N}=122)$, the proportion of patients who experienced an adverse event was lower with carvedilol CR compared with carvedilol IR (Henderson et al 2006). Moreover, headache, orthostatic hypotension, diarrhea, and dizziness occurred more frequently with carvedilol IR than with carvedilol CR (Table 1) (Henderson et al 2006). It must be acknowledged, however, that this was a small study (122 patients) of short duration (approximately 5 weeks). The adverse event profile was comparable between carvedilol IR and carvedilol $\mathrm{CR}$ among patients with heart failure or post-MI enrolled in a pharmacokinetics study, with no notable increase in events when patients were switched from carvedilol IR to CR (Packer et al 2006). In the carvedilol CR hypertension efficacy study, the occurrences of fatigue, dizziness, and headache were comparable between the combined 
Table I Treatment-related adverse events with IR and CR carvedilol ${ }^{\mathrm{a}}$

\begin{tabular}{|c|c|c|c|c|c|c|c|}
\hline \multirow[b]{3}{*}{ Variable } & \multicolumn{7}{|l|}{ Regimen } \\
\hline & \multicolumn{3}{|c|}{ Carvedilol IR ${ }^{\mathrm{b}}$ BID } & \multicolumn{4}{|c|}{ Carvedilol CR $^{\mathrm{b}}$ QD } \\
\hline & $6.25 \mathrm{mg}$ & $12.5 \mathrm{mg}$ & $25 \mathrm{mg}$ & $20 \mathrm{mg}$ & $40 \mathrm{mg}$ & $80 \mathrm{mg}$ & Placebo $^{c}$ \\
\hline Patients exposed (n) & 65 & 26 & 50 & 67 & 26 & 51 & 63 \\
\hline Patients with adverse events, $\mathrm{n}(\%)$ & $18(27.7)$ & $5(19.2)$ & $14(28.0)$ & $17(25.4)$ & I (3.8) & $7(13.7)$ & $16(25.4)$ \\
\hline \multicolumn{8}{|l|}{ Adverse event, $\mathrm{n}(\%)$} \\
\hline Headache & II (I6.9) & $3(11.5)$ & $10(20)$ & $6(9.0)$ & I (3.8) & $5(9.8)$ & 7 (II.I) \\
\hline Orthostatic hypotension & $3(4.6)$ & $2(7.7)$ & $2(4.0)$ & $3(4.5)$ & $0(0)$ & $2(3.9)$ & $5(7.9)$ \\
\hline Dizziness & $3(4.6)$ & $2(7.7)$ & $3(6.0)$ & $\mathrm{I}(\mathrm{I} .5)$ & $0(0)$ & $0(0)$ & $4(6.3)$ \\
\hline Fatigue & $0(0)$ & $0(0)$ & $0(0)$ & $\mathrm{I}(\mathrm{I} .5)$ & $0(0)$ & $0(0)$ & $2(3.2)$ \\
\hline Somnolence & $0(0)$ & $0(0)$ & I (2.0) & $2(3.0)$ & $0(0)$ & $0(0)$ & $0(0)$ \\
\hline Asthenia & $2(3.1)$ & $0(0)$ & $0(0)$ & $0(0)$ & $0(0)$ & $0(0)$ & $0(0)$ \\
\hline Dyspepsia & I (I.5) & $0(0)$ & $0(0)$ & $\mathrm{I}(\mathrm{I} .5)$ & $0(0)$ & $0(0)$ & $0(0)$ \\
\hline Edema, peripheral & $\mathrm{I}(\mathrm{I} .5)$ & $0(0)$ & $0(0)$ & $0(0)$ & $0(0)$ & I (2.0) & $0(0)$ \\
\hline Hypotension & I (I.5) & $0(0)$ & $0(0)$ & $\mathrm{I}(\mathrm{I} .5)$ & $0(0)$ & $0(0)$ & $0(0)$ \\
\hline
\end{tabular}

${ }^{a}$ Only the most commonly reported adverse events (considered by the investigator to be related to study medication) are listed.

${ }^{b}$ Adverse events attributed to any dose of carvedilol IR or carvedilol CR include events that were reported by patients in any treatment group who were receiving the designated regimen (sessions I-4), adverse events that occurred in the downtitration period are not included (session 5).

'Placebo group included the downtitration period (session 5) for the 2 low-dose groups that previously received carvedilol in sessions I-4.

Abbreviations: CR, controlled release, IR, immediate release, BID, twice daily, QD, once daily.

Reprinted from Henderson LS, Tenero DM, Baidoo CA, et al Pharmacokinetic and pharmacodynamic comparison of controlled-release carvedilol and immediate-release carvedilol at steady state in patients with hypertension, Am J Cardiol, 98: 17L-26L. Copyright @ 2006, with permission from Elsevier.

carvedilol dose groups and the placebo group (Weber et al 2006a).

\section{Potential for improving patient adherence to therapy Reducing pill burden}

Use of multiple concomitant medications in the treatment of hypertension, heart failure, and MI contributes to the polypharmacy that is common among patients receiving carvedilol. Combinations of diuretics, ACE inhibitors, digitalis, glycosides, and beta-blockers are common in the treatment regimen for patients with heart failure. After an MI, guidelines recommend treatment with multiple evidence-based medications, including aspirin, clopidogrel, a statin, an ACE inhibitor, and a beta-blocker. In the field of hypertension, few patients achieve adequate blood pressure control on monotherapy (Bangalore et al 2007a). In fact, in ALLHAT, a mean of 2 drugs was required to reach blood pressure targets (Cushman et al 2002). Couple these findings with the treatment regimens for common comorbidities (eg, diabetes, coronary artery disease), and the pill burden can become quite substantial.

Both the number of pills taken daily and frequency of dosing affect patient adherence to prescribed therapies, and adherence, in turn, influences clinical outcomes (Claxton et al 2001). In patients with hypertension, lack of adherence has been shown to reduce treatment benefit and increase the risk of poor treatment outcome (Frishman 2007b). Among patients post-MI, lack of adherence to statins, calcium channel blockers, and beta-blockers significantly increased mortality risk ( $\mathrm{p}=0.001)$ (Rasmussen et al 2007).

One method for reducing pill burden is to design drugs that can be dosed less frequently. One such example is carvedilol CR. Once-daily dosing has been shown to improve adherence (Frishman 2007b). Data compiled from 76 studies covering a range of therapeutic areas demonstrated that once-daily dosing is associated with the highest adherence rate among various dosing regimens (Claxton et al 2001). The levels of adherence declined as the number of daily doses increased ( $79 \%$ for once daily, $69 \%$ for twice daily, $65 \%$ for 3 times daily, and 51\% for 4 times daily). Moreover, the adherence benefit of once-daily dosing has been specifically observed with a beta-blocker (metoprolol) used for the treatment of hypertension (Baird et al 1984).

The Compliance and Quality of Life Study Comparing Once-Daily Controlled-Release Carvedilol CR and TwiceDaily Immediate-Release Carvedilol IR in Patients with Heart Failure (CASPER) trial was based on the assumptions that adherence to the medication regimen would be 
$75 \%$ with twice-daily medication and $90 \%$ with once-daily medication (Hauptman et al 2006). Patients with mild to severe heart failure and a left ventricular ejection fraction of $40 \%$ or less were randomized to either a double-blind arm (morning carvedilol CR plus an evening placebo versus morning and evening carvedilol IR) or to an open-label arm (carvedilol CR). However, preliminary results demonstrated no difference in patient adherence between once-daily and twice-daily regimens (88\% versus 89\%) (Udelson et al 2007; Carter et al 2008). These results may be explained, in part, by the consideration that patient adherence may be higher in clinical trials than in the community setting. Indeed, the carvedilol IR recipients in this trial had a higher than anticipated compliance rate.

Another option for reducing pill burden is to reduce the number of individual pills through use of a fixed-dose combination (FDC). Fixed-dose combinations have become increasingly common in the field of hypertension because of the frequent need for multiple concomitant antihypertensives to control blood pressure. Fixed-dose combinations may also work synergistically, resulting in a lower total dose of each individual drug (Rao et al 1998). The ability to reduce drug doses can also reduce the risk of side effects. Use of an FDC may also have positive effects on patient adherence. In a study comparing adherence between patients treated with an FDC $(n=11,925)$ and patients treated with a free-drug regimen $(n=8317)$, those receiving an FDC had a significantly decreased risk of nonadherence $(p<0.0001)$ (Bangalore et al 2007a).

An FDC consisting of carvedilol and the ACE inhibitor lisinopril is currently in development. The Coreg and Lisinopril Combination Therapy in Hypertensive Subjects (COSMOS) trial is assessing the efficacy and safety of a carvedilol/lisinopril FDC (ClinicalTrials.gov Identifier: NCT00347360). COSMOS is a randomized, double-blind, double-dummy, parallel-group trial employing a $4 \times 4$ factorial design that will evaluate carvedilol $(20,40$, or $80 \mathrm{mg}$ daily) alone, lisinopril (10, 20, or $40 \mathrm{mg}$ daily) alone, and all permutations of the combined drugs in patients with hypertension.

\section{Quality of life and patient satisfaction}

Quality of life is more difficult to assess than concrete measures such as blood pressure or mortality. If improved tolerability and reduced risk for side effects were considered, one would anticipate an improvement in this category with carvedilol as appraised relative to traditional betablockers.
Some clinical trials have revealed improvements in patient-reported quality-of-life assessments. The effects of carvedilol IR on patient global assessment were reported in patients with heart failure (Packer et al 2002). In a study of 2289 patients with heart failure, patients receiving treatment with carvedilol were more likely to show moderate or marked improvement and less likely to show moderate or marked worsening versus placebo. In the previously described COMET study, a patient-completed, self-assessment scoring system that was evaluated every 4 months of treatment demonstrated a significantly improved self-score for patients treated with carvedilol $(p<0.0068)$ (Cleland et al 2006).

A prespecified secondary analysis of the GEMINI study used the Diabetes Symptom Checklist to record patient-reported occurrences of diabetes-related symptoms in 8 domains, namely psychology (fatigue), psychology (cognitive), neuropathy (pain), neuropathy (sensory), cardiology, ophthalmology, hyperglycemia, and hypoglycemia (McGill et al 2007). After 5 months of treatment, the results significantly favored carvedilol over metoprolol tartrate in overall maintenance score $(p=0.02)$, hypoglycemia symptoms $(\mathrm{p}=0.02)$, and hyperglycemia symptoms $(\mathrm{p}=0.005)$.

\section{Conclusions}

The varied mechanisms of action of carvedilol allow it to be an effective treatment of hypertension, heart failure, and post-MI LVD, with less of the metabolic and tolerability concerns associated with traditional beta-blockers. The key difference from the majority of beta-blockers appears to be vasodilation mediated by alpha 1-adrenergic receptor blockade, which decreases total peripheral resistance (Pedersen et al 2007). Furthermore, carvedilol's antioxidant and anti-inflammatory effects may provide additional cardioprotective effects beyond that of traditional beta-blockers (Dandona et al 2007). Carvedilol has a long history and a proven track record in the treatment of hypertension and cardiac dysfunction. The once-daily formulation of carvedilol provides a more convenient option and may confer an adherence benefit over the twice-daily formulation in conditions traditionally prone to noncompliance such as hypertension and post-MI LVD.

\section{Acknowledgments}

The authors would like to thank Tamalette Loh, PhD, ProEd Communications, Inc. ${ }^{\circledR}$, for her medical editorial assistance with this manuscript. 


\section{Disclosures}

The authors have no conflicts of interest to disclose.

\section{References}

Al-Hesayen A, Azevedo ER, Floras JS, et al. 2005. Selective versus nonselective beta-adrenergic receptor blockade in chronic heart failure: differential effects on myocardial energy substrate utilization. Eur $J$ Heart Fail, 7:618-23.

American Heart Association. 2007. Heart Disease and Stroke Statistics - 2007 Update. Dallas, Texas: American Heart Association.

August P. 2003. Initial treatment of hypertension. $N$ Engl J Med, 348:610-7.

Australia/New Zealand Heart Failure Research Collaborative Group. 1997. Randomised, placebo-controlled trial of carvedilol in patients with congestive heart failure due to ischaemic heart disease. Australia/ New Zealand Heart Failure Research Collaborative Group. Lancet, 349:375-80.

Baird MG, Bentley-Taylor MM, Carruthers SG, et al. 1984. A study of efficacy, tolerance and compliance of once-daily versus twice-daily metoprolol (Betaloc) in hypertension. Betaloc Compliance Canadian Cooperative Study Group. Clin Invest Med, 7:95-102.

Bakris GL, Fonseca V, Katholi RE, et al. 2004. Metabolic effects of carvedilol vs metoprolol in patients with type 2 diabetes mellitus and hypertension: a randomized controlled trial. JAMA, 292:2227-36.

Bakris GL, Hart P, Ritz E. 2006a. Beta blockers in the management of chronic kidney disease. Kidney Int, 70:1905-13.

Bakris GL, Tarka EA, Waterhouse B, et al. 2006b. Cardiovascular risk factors in hypertension: rationale and design of studies to investigate the effects of controlled-release carvedilol on regression of left ventricular hypertrophy and lipid profile. Am J Cardiol, 98:46L-52L.

Bangalore S, Kamalakkannan G, Parkar S, et al. 2007a. Fixed-dose combinations improve medication compliance: a meta-analysis. Am J Med, 120:713-9.

Bangalore S, Messerli FH, Kostis JB, et al. 2007b. Cardiovascular protection using beta-blockers: a critical review of the evidence. $J$ Am Coll Cardiol, 50:563-72.

Basat O, Ucak S, Seber S, et al. 2006. After myocardial infarction carvedilol improves insulin resistance compared to metoprolol. Clin Res Cardiol, 95:99-104.

Carter NJ, Keating GM. 2008. Controlled-release carvedilol. Am J Cardiovasc Drugs, 8:271-82.

Chobanian AV, Bakris GL, Black HR, et al. 2003. Seventh report of the Joint National Committee on Prevention, Detection, Evaluation, and Treatment of High Blood Pressure. Hypertension, 42:1206-52.

Claxton AJ, Cramer J, Pierce C. 2001. A systematic review of the associations between dose regimens and medication compliance. Clin Ther, 23:1296-310.

Cleland JG, Charlesworth A, Lubsen J, et al. 2006. A comparison of the effects of carvedilol and metoprolol on well-being, morbidity, and mortality (the "patient journey") in patients with heart failure: a report from the Carvedilol Or Metoprolol European Trial (COMET). J Am Coll Cardiol, 47:1603-11.

Colagiuri S, Cull CA, Holman RR. 2002. Are lower fasting plasma glucose levels at diagnosis of type 2 diabetes associated with improved outcomes? UK prospective diabetes study 61. Diabetes Care, 25:1410-7.

Cushman WC, Ford CE, Cutler JA, et al. 2002. Success and predictors of blood pressure control in diverse North American settings: the antihypertensive and lipid-lowering treatment to prevent heart attack trial (ALLHAT). J Clin Hypertens (Greenwich), 4:393-404.

Dandona P, Ghanim H, Brooks DP. 2007. Antioxidant activity of carvedilol in cardiovascular disease. J Hypertens, 25:731-41.

Dargie HJ. 2001. Effect of carvedilol on outcome after myocardial infarction in patients with left-ventricular dysfunction: the CAPRICORN randomised trial. Lancet, 357:1385-90.
Doughty RN, Whalley GA, Walsh HA, et al. 2004. Effects of carvedilol on left ventricular remodeling after acute myocardial infarction: the CAPRICORN Echo Substudy. Circulation, 109:201-6.

Egan BM, Basile J, Chilton RJ, et al. 2005. Cardioprotection: the role of beta-blocker therapy. J Clin Hypertens (Greenwich), 7:409-16.

Fonarow GC. 2004. Managing the patient with diabetes mellitus and heart failure: issues and considerations. Am J Med, 116(Suppl $5 \mathrm{~A}): 76 \mathrm{~S}-88 \mathrm{~S}$.

Fonarow GC. 2006. Profile of carvedilol controlled-release: a new once-daily formulation of carvedilol. Expert Opin Pharmacother, 7:2533-46.

Fonarow GC, Lukas MA, Robertson M, et al. 2007. Effects of carvedilol early after myocardial infarction: analysis of the first 30 days in Carvedilol Post-Infarct Survival Control in Left Ventricular Dysfunction (CAPRICORN). Am Heart J, 154:637-44.

Frishman WH. 1998. Carvedilol. N Engl J Med, 339:1759-65.

Frishman WH. 2003. Alpha- and beta-adrenergic blocking drugs. In: Frishman WH, Sonnenblick EH, Sica DA (eds). Cardiovascular pharmacotherapeutics. 2nd ed. New York: McGraw Hill. p 67-97.

Frishman WH. 2007a. A historical perspective on the development of betaadrenergic blockers. J Clin Hypertens, 9(4 Suppl 3):19-27.

Frishman WH. 2007b. Importance of medication adherence in cardiovascular disease and the value of once-daily treatment regimens. Cardiol Rev, 15:257-63.

Giugliano D, Acampora R, Marfella R, et al. 1997. Metabolic and cardiovascular effects of carvedilol and atenolol in non-insulin-dependent diabetes mellitus and hypertension. A randomized, controlled trial. Ann Intern Med, 126:955-9.

Goldhammer E, Maor I, Shnitzer S, et al. 2007. The early anti-oxidant effect of carvedilol predicts the clinical course in congestive heart failure patients. J Cardiovasc Med (Hagerstown), 8:453-6.

Greenberg BH, Mehra M, Teerlink JR, et al. 2006. COMPARE: comparison of the effects of carvedilol CR and carvedilol IR on left ventricular ejection fraction in patients with heart failure. Am J Cardiol, 98:53L-9L.

Hauptman PJ, Pressler SJ, Sackner-Bernstein J, et al. 2006. Rationale and design of CASPER: compliance and quality of life study comparing once-daily carvedilol CR and twice-daily carvedilol IR in patients with heart failure. Am J Cardiol, 98:60L-6L.

Henderson LS, Tenero DM, Baidoo CA, et al. 2006. Pharmacokinetic and pharmacodynamic comparison of controlled-release carvedilol and immediate-release carvedilol at steady state in patients with hypertension. Am J Cardiol, 98:17L-26L.

Henderson LS, Tenero DM, Campanile AM, et al. 2007. Ethanol does not alter the pharmacokinetic profile of the controlled-release formulation of carvedilol. J Clin Pharmacol, 47:1358-65.

Jacob S, Rett K, Wicklmayr M, et al. 1996. Differential effect of chronic treatment with two beta-blocking agents on insulin sensitivity: the carvedilol-metoprolol study. J Hypertens, 14:489-94.

Johnson ML, Pietz K, Battleman DS, et al. 2004. Prevalence of comorbid hypertension and dyslipidemia and associated cardiovascular disease. Am J Manag Care, 10:926-32.

Kozlovski VI, Lomnicka M, Chlopicki S. 2006. Nebivovol and carvedilol induce NO-dependent coronary vasodilatation that is unlikely to be mediated by extracellular ATP in the isolated guinea pig heart. Pharmacol Rep, 58(Suppl):103-10.

Kurum T, Tatli E, Yuksel M. 2007. Effects of carvedilol on plasma levels of pro-inflammatory cytokines in patients with ischemic and nonischemic dilated cardiomyopathy. Tex Heart Inst J, 34:52-9.

Lefkowitz RJ, Rajagopal K, Whalen EJ. 2006. New roles for beta-arrestins in cell signaling: not just for seven-transmembrane receptors. Mol Cell, $24: 643-52$.

Leventhal MJ, Riegel B, Carlson B, et al. 2005. Negotiating compliance in heart failure: remaining issues and questions. Eur J Cardiovasc Nurs, 4:298-307.

Lindholm LH, Carlberg B, Samuelsson O. 2005. Should beta blockers remain first choice in the treatment of primary hypertension? A metaanalysis. Lancet, 366:1545-53. 
Maack C, Cremers B, Flesch M, et al. 2000. Different intrinsic activities of bucindolol, carvedilol and metoprolol in human failing myocardium. Br J Pharmacol, 130:1131-9.

Maggi E, Marchesi E, Covini D, et al. 1996. Protective effects of carvedilol, a vasodilating beta-adrenoceptor blocker, against in vivo low density lipoprotein oxidation in essential hypertension. $J$ Cardiovasc Pharmacol, 27:532-8.

Manley S. 2003. Haemoglobin A1c - a marker for complications of type 2 diabetes: the experience from the UK Prospective Diabetes Study (UKPDS). Clin Chem Lab Med, 41:1182-90.

McGill JB, Bakris GL, Fonseca V, et al. 2007. beta-Blocker use and diabetes symptom score: results from the GEMINI study. Diabetes Obes Metab, 9:408-17.

McMurray J, Kober L, Robertson M, et al. 2005. Antiarrhythmic effect of carvedilol after acute myocardial infarction: results of the Carvedilol Post-Infarct Survival Control in Left Ventricular Dysfunction (CAPRICORN) trial. $J$ Am Coll Cardiol, 45:525-30.

Messerli FH, Grossman E. 2004. beta-Blockers in hypertension: is carvedilol different? Am J Cardiol, 93:7B-12B

Metra M, Giubbini R, Nodari S, et al. 2000. Differential effects of betablockers in patients with heart failure: A prospective, randomized, double-blind comparison of the long-term effects of metoprolol versus carvedilol. Circulation, 102:546-51.

Moreno AJ, Santos DJ, Palmeira CM. 1998. Ischemic heart disease: the role of mitochondria - carvedilol prevents lipid peroxidation of mitochondrial membranes. Rev Port Cardiol, 17(Suppl 2):II63-77.

Nakamura K, Kusano K, Nakamura Y, et al. 2002. Carvedilol decreases elevated oxidative stress in human failing myocardium. Circulation, 105:2867-71.

Nichols AJ, Sulpizio AC, Ashton DJ, et al. 1989. The interaction of the enantiomers of carvedilol with alpha 1- and beta 1-adrenoceptors. Chirality, 1:265-70.

Othman AA, Tenero DM, Boyle DA, et al. 2007. Population pharmacokinetics of S(-)-carvedilol in healthy volunteers after administration of the immediate-release (IR) and the new controlled-release (CR) dosage forms of the racemate. Aaps $J, 9: \mathrm{E} 208-18$.

Otterstad JE, Ford I. 2002. The effect of carvedilol in patients with impaired left ventricular systolic function following an acute myocardial infarction. How do the treatment effects on total mortality and recurrent myocardial infarction in CAPRICORN compare with previous betablocker trials? Eur J Heart Fail, 4:501-6.

Packer M, Bristow MR, Cohn JN, et al. 1996. The effect of carvedilol on morbidity and mortality in patients with chronic heart failure. US Carvedilol Heart Failure Study Group. N Engl J Med, 334:1349-55.

Packer M. 1998. Beta-adrenergic blockade in chronic heart failure: principles, progress, and practice. Prog Cardiovasc Dis, 41:39-52.

Packer M, Fowler MB, Roecker EB, et al. 2002. Effect of carvedilol on the morbidity of patients with severe chronic heart failure: results of the carvedilol prospective randomized cumulative survival (COPERNICUS) study. Circulation, 106:2194-9.

Packer M, Lukas MA, Tenero DM, et al. 2006. Pharmacokinetic profile of controlled-release carvedilol in patients with left ventricular dysfunction associated with chronic heart failure or after myocardial infarction. Am J Cardiol, 98:39L-45L.

Padi SS, Chopra K. 2002. Salvage of cyclosporine A-induced oxidative stress and renal dysfunction by carvedilol. Nephron, 92:685-92.

Patel PA, Tilley DG, Rockman HA. 2008. Beta-Arrestin-Mediated Signaling in the Heart. Circ J, 72:1725-9.

Pedersen ME, Cockcroft JR. 2007. The vasodilatory beta-blockers. Curr Hypertens Rep, 9:269-77.

Podbregar M, Voga G. 2002. Effect of selective and nonselective betablockers on resting energy production rate and total body substrate utilization in chronic heart failure. J Card Fail, 8:369-78.

Poole-Wilson PA, Swedberg K, Cleland JG, et al. 2003. Comparison of carvedilol and metoprolol on clinical outcomes in patients with chronic heart failure in the Carvedilol Or Metoprolol European Trial (COMET): randomised controlled trial. Lancet, 362:7-13.
Rao RB, Goldfrank LR. 1998. Fixed-dose combination therapy: panacea or poison? Intensive Care Med, 24:283-5.

Rasmussen JN, Gislason GH, Rasmussen S, et al. 2007. Use of statins and beta-blockers after acute myocardial infarction according to income and education. J Epidemiol Community Health, 61:1091-7.

Rosamond W, Flegal K, Furie K, et al. 2008. Heart disease and stroke statistics - 2008 update: a report from the American Heart Association Statistics Committee and Stroke Statistics Subcommittee. Circulation, 117:e25-146.

Stamler J, Wentworth D, Neaton JD. 1986. Prevalence and prognostic significance of hypercholesterolemia in men with hypertension. Prospective data on the primary screenees of the Multiple Risk Factor Intervention Trial. Am J Med, 80:33-9.

Stanley WC, Recchia FA, Lopaschuk GD. 2005. Myocardial substrate metabolism in the normal and failing heart. Physiol Rev, 85:1093-129.

Sundberg S, Tiihonen K, Gordin A. 1987. Vasodilatory effects of carvedilol and pindolol. J Cardiovasc Pharmacol, 10(Suppl 11):S76-80.

Tatli E, Kurum T. 2005. A controlled study of the effects of carvedilol on clinical events, left ventricular function and proinflammatory cytokines levels in patients with dilated cardiomyopathy. Can $J$ Cardiol, 21:344-8.

Tenero DM, Henderson LS, Baidoo CA, et al. 2006. Pharmacokinetic properties of a new controlled-release formulation of carvedilol. $\mathrm{Am} J$ Cardiol, 98:5L-16L.

Toda N. 2003. Vasodilating beta-adrenoceptor blockers as cardiovascular therapeutics. Pharmacol Ther, 100:215-34.

Torp-Pedersen C, Poole-Wilson PA, Swedberg K, et al. 2005. Effects of metoprolol and carvedilol on cause-specific mortality and morbidity in patients with chronic heart failure - COMET. Am Heart $J$, 149:370-6.

Torp-Pedersen C, Metra M, Charlesworth A, et al. 2007. Effects of metoprolol and carvedilol on pre-existing and new onset diabetes in patients with chronic heart failure: data from the Carvedilol Or Metoprolol European Trial (COMET). Heart, 93:968-73.

Udelson JE, Pressler SJ, Sackner-Bernstein J, et al. 2007. Compliance with Once Daily Controlled Release vs Twice Daily Immediate Release Carvedilol in Patients with Heart Failure: The CASPER Trial $J$ Card Fail, 13:S135

Vittorio TJ, Zolty R, Kasper ME, et al. 2008. Differential effects of carvedilol and metoprolol succinate on plasma norepinephrine release and peak exercise heart rate in subjects with chronic heart failure. J Cardiovasc Pharmacol Ther, 13:51-7.

Wallhaus TR, Taylor M, DeGrado TR, et al. 2001. Myocardial free fatty acid and glucose use after carvedilol treatment in patients with congestive heart failure. Circulation, 103:2441-6.

Weber MA, Bakris GL, Tarka EA, et al. 2006a. Efficacy of a once-daily formulation of carvedilol for the treatment of hypertension. $J$ Clin Hypertens (Greenwich), 8:840-9.

Weber MA, Sica DA, Tarka EA, et al. 2006b. Controlled-release carvedilol in the treatment of essential hypertension. Am J Cardiol, 98:32L-8L

Wisler JW, DeWire SM, Whalen EJ, et al. 2007. A unique mechanism of beta-blocker action: carvedilol stimulates beta-arrestin signaling. Proc Natl Acad Sci U S A, 104:16657-62.

World Health Organization. 2007. Cardiovascular diseases. Fact sheet no. 317 [online]. Accessed August 4, 2008. URL: http://www.who. int/mediacentre/factsheets/fs317/en/index.html.

Wright JT Jr, Bakris GL, Bell DS, et al. 2007. Lowering blood pressure with beta-blockers in combination with other renin-angiotensin system blockers in patients with hypertension and type 2 diabetes: results from the GEMINI Trial. J Clin Hypertens (Greenwich), 9:842-9.

Yang SP, Ho LJ, Cheng SM, et al. 2004. Carvedilol differentially regulates cytokine production from activated human peripheral blood mononuclear cells. Cardiovasc Drugs Ther, 18:183-8. 